\title{
Bacteria Hunt
}

\section{Evaluating multi-paradigm BCI interaction}

\author{
Christian Mühl · Hayrettin Gürkök • Danny Plass-Oude Bos • Marieke E. Thurlings • \\ Lasse Scherffig • Matthieu Duvinage • Alexandra A. Elbakyan • SungWook Kang • \\ Mannes Poel · Dirk Heylen
}

Received: 26 February 2010 / Accepted: 24 July 2010 / Published online: 14 August 2010

(C) The Author(s) 2010. This article is published with open access at Springerlink.com

\begin{abstract}
The multimodal, multi-paradigm brain-computer interfacing (BCI) game Bacteria Hunt was used to evaluate two aspects of $\mathrm{BCI}$ interaction in a gaming context. One goal was to examine the effect of feedback on the ability of the user to manipulate his mental state of relaxation. This was done by having one condition in which the subject played the game with real feedback, and another with sham feedback. The feedback did not seem to affect the game experience (such as sense of control and tension) or the objective indicators of relaxation, alpha activity and heart rate. The results are discussed with regard to clinical neurofeedback studies. The second goal was to look into possible interactions between the two BCI paradigms used in the game: steady-state visually-evoked potentials (SSVEP) as an indicator of concentration, and alpha activity as a measure of
\end{abstract}

C. Mühl ( $\varangle) \cdot$ H. Gürkök · D. Plass-Oude Bos · M. Poel ·

D. Heylen

University of Twente, Enschede, The Netherlands

e-mail: muehlc@cs.utwente.nl

M.E. Thurlings

TNO Human Factors, Soesterberg, The Netherlands

M.E. Thurlings

Utrecht University, Utrecht, The Netherlands

L. Scherffig

Academy of Media Arts Cologne, Cologne, Germany

M. Duvinage

University of Mons, Mons, Belgium

A.A. Elbakyan

Kazakh National Technical University, Almaty, Kazakhstan

S.W. Kang

Gwangju Institute of Science and Technology, Gwangju,

South Korea relaxation. SSVEP stimulation activates the cortex and can thus block the alpha rhythm. Despite this effect, subjects were able to keep their alpha power up, in compliance with the instructed relaxation task. In addition to the main goals, a new SSVEP detection algorithm was developed and evaluated.

Keywords Brain-computer interfacing · Multimodal interaction $\cdot$ Steady-state visually-evoked potentials . Concentration · Neurofeedback $\cdot$ Relaxation $\cdot$ Game

\section{Introduction}

1.1 BCI and multimodal interaction

The multidisciplinary study of brain-computer interfaces (BCI) combines fields including but not limited to engineering, cognitive neuroscience, psychology, machine learning and human-computer interaction. The fields of applications using this direct communication channel from brain to machine are just as diverse, from rehabilitation to affective computing.

Where originally BCI research was focused on paralysed patients, new developments such as affordable and wireless dry cap technology make BCI viable for healthy users. Besides the novelty factor and the magic of being able to use your brain directly for control, BCI also provides private, hands-free interaction. It increases the information users can provide to applications and applications in turn can react more appropriately, taking the user's mental state into account [11].

A large potential target group are gamers, as games are an area where novelty is appreciated and learning new skills is often part of the challenge [35]. Games can also be a very interesting option for research with patients. Virtual worlds 
can provide a safe environment to learn to produce specific brain activity: accidentally steering your wheel chair off the stairs is less painful in a virtual environment. Additionally, the game element can ensure that tedious training is fun and the patients stay motivated.

Unfortunately, there are still issues that cause problems when trying to use a $\mathrm{BCI}$. There are large differences in the brain activity between people, and even within one person the brain activity changes quickly over time [8]. This makes it difficult to create a system that will understand what the user is trying to do, especially for a longer duration. Depending on the BCI paradigm used a lot of training may be required (ranging from minutes to months) before the user is able to generate the correct signal for the system. Alternatively, the system may be trained with user specific data to recognise the user's brain activity associated with a certain (mental) action. Neither method may work if the person trying to use the system falls into the category of the socalled BCI illiterate like $20 \%$ of the population [34]. This means that this user may not be able to generate the signal in a way that is measurable by the system, and hence will not be able to control it. Moreover, as a result of using electroencephalography (EEG) as a measurement method, the recorded brain activity has a low signal-to-noise ratio and is susceptible to eye or muscle artefacts. These problems cause a high level of uncertainty when analysing and interpreting brain signals. There are also issues with speed and timing, as relevant brain activity needs to be induced, recorded, analysed, and interpreted before the corresponding action can be performed in the application.

Although paralysed patients, for whom BCI is the only interaction modality left, may be willing to accept resulting problems with robustness and speed, healthy subjects will be less forgiving. For them, many other input modalities are available. Therefore, the traditional usability and user experience challenges have to be solved as well, in order to exploit the full potential of this innovative technology and to increase its acceptance among the general population [39].

One possibility to attenuate the impact of the above mentioned limitations of BCI on the usability, is to apply it in combination with traditional control modalities. This way one can use its advantages, while at the same time compensating for the limitations and downsides of the new interaction mode. Furthermore, several types (paradigms) of BCI might be combined as well to increase the bandwidth of interaction.

However, current BCI research applications mostly restrict themselves to the use of one modality (BCI) and one BCI paradigm, to control one type of interaction in a highly simplified game [41]. It is a big step from this situation to the interaction found in current commercial video games, which might provide a gold standard to test the applicability of new modalities for interaction, such as BCI. Besides the large number of actions that can be carried out in game worlds nowadays, gamers will not behave according to the restrictions often applied in current BCI research in order to reduce artefacts in EEG. Gamers will move, execute multiple tasks at once, and use multiple interaction modalities in parallel.

A BCI game was developed and implemented [30] to address the use of BCI in addition to traditional input modalities and the simultaneous utilisation of multiple types of BCI. This game provides an environment which enables the research on multimodal, multiparadigm BCI under ecologically valid conditions, that is in an authentic, potentially multi-player, computer game setting. Thereby it is supposed to bridge the gap between controlled laboratory experiments and competitive and artefact-prone real-world applications.

More specifically, the game combines the interaction via two varieties of $\mathrm{BCI}$ in addition to the interaction via keyboard to manipulate a game world within a simple game concept, that is the hunt for objects on a $2 \mathrm{D}$ plane. One type of BCI, a neurofeedback-like approach, is supposed to reward the user with a better chance to gain points for the maintenance of a high state of relaxation. The second type, using steady-state visually evoked potentials (SSVEP), evaluates the user's concentration on a visual game element.

The current study uses the game as a platform to specifically explore two research questions associated with the use of multiple types of $\mathrm{BCI}$ in addition to traditional modes of input. The first goal is the assessment of the efficacy of the neurofeedback-like BCI as a game element via subjective and objective indicators. Does the reward of a specific mental state, relaxation, lead to an increase of this state? The second goal is to examine possible interactions between the different BCIs implemented. Will the simultaneous use of multiple types of BCIs result in mutual interference? Furthermore, for the second type of $\mathrm{BCI}$ a new detection algorithm will be proposed and tested.

In Sect. 2 the reader will be introduced to some of the different types of BCIs available, and in more detail to the types of BCIs used in the current version of the game. In Sect. 3 the system, including game design and BCI processing, will be described together with the proposed SSVEP detection algorithm. Then the specific experimental hypotheses and methods will be outlined. In Sect. 5 the results will be described, which are then discussed in Sect. 6.

\section{Types of BCI}

There are several types of BCIs. In the following, a twodimensional classification of some of these BCI paradigms is given. It is derived from the 3 category classification presented in [57].

The dimensions of this classification are defined by (i) the dependence on external stimuli and (ii) the dependence on 


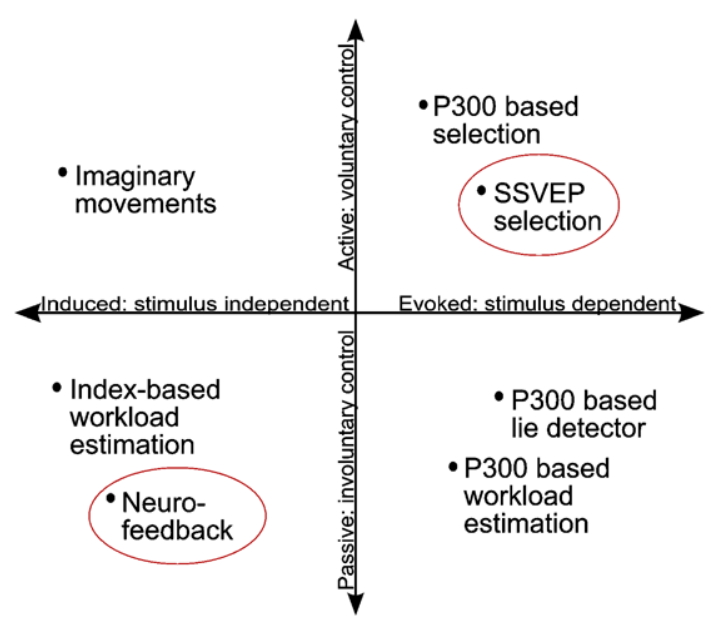

Fig. 1 A classification of BCI paradigms, spanning voluntariness (passive vs. active) and stimulus dependency (user induced vs. stimulus evoked). The applied paradigms are circled in red

an intention to create a neural activity pattern as illustrated in Fig. 1.

Axis (i) stretches from exogenous (or evoked) to endogenous (or induced) input. The former covers all forms of BCI which necessarily presuppose an external stimulus. Steadystate visually-evoked potentials as neural correlates of stimulus frequencies, for instance, may be detected if and only if evoked by a stimulus. They are therefore a clear example of exogenous input. Endogenous input, on the other hand, does not presuppose an external stimulus. An example is the use of alpha band power in neurofeedback applications. While alpha activity may be influenced by external stimuli, in principle it can be measured when no stimulus is present and is therefore classified as endogenous. Another way of separating both poles of the axis is the possibility of building a selfpaced (or asynchronous) BCI [45]: only endogenous input can be used to build a system that is self-paced, in the sense that it can decide at all times whether a user has initiated an action by brain activity. On the other hand, all forms of exogenous BCI are necessarily synchronous.

Axis (ii) stretches from active to passive input. Active input presupposes an intention to control brain activity while passive input does not. Imagined movements, for instance, can only be detected when subjects intend to perform these, making the paradigm a prototypical application of active BCI. Alpha power and other indicators of mental state, on the other hand, can also be measured when subjects do not exhibit an intention to produce it.

For the game developed, we decided to forgo paradigms that need extensive user training or machine learning, while at the same time we wanted a variety of different types of paradigms. This resulted in the selection of a neurofeedback paradigm based on the power in the alpha band and steady-state visually-evoked potentials, both introduced in detail below.

\subsection{Alpha neurofeedback}

In the current study, the aim was the incorporation of BCI as an auxiliary control modality that is supposed to have a positive effect on the state of the user. The user controlled a game element by means of his relaxation. During the game relaxation should be a desirable state, as it is indirectly rewarded with advantages in game play. This approach can be described with the concept of a biocybernetic feedback loop [11] and was before implemented in the simple game "Brainball" [19]. The application is reacting to the observed physiological indicator of relaxation, resulting in feedback of the state to the user. This feedback functions as a reminder and bias to the user to maintain or deepen the state of relaxation.

A conceptually similar approach can be found in neurofeedback studies. Neurofeedback is a variant of biofeedback that enables the user to monitor and regulate body functions that are normally not accessible to him. Specifically, indicators of brain activity are made observable via visualisation or sonification. Examples of such indicators are the sensorymotor rhythm (8-15 Hz measured over central regions), alpha band power (8-12 Hz measured over parietal regions), or slow cortical potentials. The potential areas of the application of neurofeedback are manifold, and can be found in therapeutical contexts as well as in every-day uses. Prominent current applications involve the treatment of attentiondeficit epilepsy [52], hyperactivity disorder [4] and of substance abuse [51]. In recent years studies also revealed performance increases in healthy subjects as a result of certain neurofeedback protocols [14]. The rationale for these procedures is the assumption that training subjects to increase activation in relevant brain areas will improve the performance.

We chose the feedback of alpha activity over parietal regions for its supposed relation with a state of relaxed wakefulness [5, 6, 36]. Alpha activity used to be considered as inversely related to neuronal activity. This can be interpreted either as cortical inhibition, or cortical idling [38]. The view that alpha power is inversely correlated to cortical activity is starting to shift, as alpha is now seen to have functional correlates to sensory processing, movement and memory [24, $37,48]$. However, the alpha rhythm is "blocked or attenuated by attention, especially visual, and mental effort" [49], further indicating its role as a correlate of mental activity. Furthermore, the feedback of alpha activity is considered to be an important tool for the treatment of stress-related anxiety disorders $[15,28]$.

\subsection{Steady-state visually-evoked potentials}

SSVEPs can be induced when a person is attending to a flickering visual stimulus, such as an LED. The frequency 
of the attended (and fixated) stimulus [40], as well as its harmonics [33], can then be traced in the EEG over the visual cortex, that is electrode locations $\mathrm{O} 1, \mathrm{Oz}$ and $\mathrm{O} 2$ according to the 10-20 system. If several stimuli are flickering with a different frequency, then the attended frequency will dominate over the other presented frequencies in the observers EEG [50].

A commonly used method to detect SSVEPs is to apply a Fast Fourier Transformation on the EEG and compare the amplitudes in the frequency bins corresponding to the frequencies of the stimuli provided. If only one stimulus is used, the amplitude of the corresponding frequency bin is compared to a set threshold. Frequencies of stimuli between 5-20 Hz elicit the highest SSVEPs [18].

SSVEPs depend on gaze, which means that users have to move their eyes or head to control a SSVEP-BCI with maximal efficiency. Covert attention to SSVEP-stimuli was shown to still allow the elicitation of SSVEPs [2, 23, 29, 32]. However, when the stimulus is presented at an angle larger than three degrees from fixation, the detection of SSVEP (and therefore also a covert attention SSVEP-BCI) becomes unlikely [53, 54].

\section{System design}

Bacteria Hunt is a alpha neurofeedback and SSVEP based experimental game. It includes, among others, a game and EEG processing component. The first component is for delivering the experimental stimuli, while the latter is for analysing the EEG signals and feeding control signals back to the game. This section provides the details of the implementation of these components and also proposes and evaluates the performance of a new SSVEP detection algorithm.

\subsection{Game design}

The game was built using the Game Maker ${ }^{\mathrm{TM}}$ development platform. It is based on the prototype described in [30]. In the following, those features that are of importance for this study are described. Following the terminology of Aarseth [1], the game can be decomposed into the game world (consisting of the objects that comprise the game) and its rules (defining the possible interactions of these objects).

\subsubsection{Game world}

The game world, as depicted in Fig. 2, consists of several entities: the player avatar (the amoeba), targets (bacteria), a numeric representation of the points obtained so far, a graph depicting the recent history of alpha band power and SSVEP classification, and a SSVEP stimulus (which is always associated with one of the target items) when it is triggered. The numeric representation of points functions as a high-level

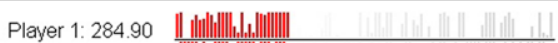

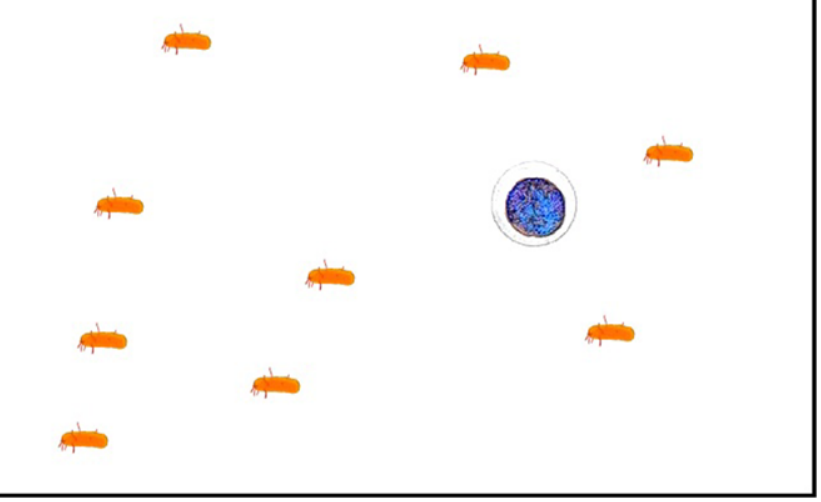

Fig. 2 The game world. Nine targets (orange "bacteria") and one player avatar (blue "amoeba") are present. The player's score is shown at the top left. The histogram above the line depicts her recent alpha band power, below the line the SSVEP classification results are marked

indicator of progress in terms of the game rules, while the graph depicting alpha band power and SSVEP classification functions as a low-level indicator of the neurophysiological coupling of the player and the game.

\subsubsection{Game rules}

For experimental reasons, three levels of the game were created as summarised in Table 1. Each level had two difficulty conditions: easy and difficult. Some general rules are the same for all levels of the game, others vary with the specific level.

General rules Movement of the player avatar is performed using the arrow keys, resulting in direct feedback through changed avatar position. Avatar position also jitters by some random noise in order to create a dynamic feel of a moving "amoeba". The game world always contains nine nonoverlapping targets. If the distance between the center of the avatar and that of a target is below a threshold radius, then the target can be "eaten". Targets disappear when eaten and are replaced by a new target, randomly placed on a free space in the game world. Successful eating results in positive points, while eating failures result in negative points. The ultimate goal of the game is to obtain as many points as possible.

Rules for the flight of targets Similar to the avatar, each target moves randomly within a short range, creating the feel of a dynamic game world. In addition, as the avatar approaches a target, the target flees. This flight behaviour depends on some factors. Levels $K A$ and $K A S$ employ alpha neurofeedback, which inversely affects target flight. Targets flee further if the scaled alpha power $\left(P_{s c a}\left(f_{\alpha}\right)\right.$, see Sect. 3.2) is 
Table 1 Game levels played during experiments

\begin{tabular}{lll}
\hline Level & Flight behaviour of bacteria & Eating of the amoeba \\
\hline Keyboard only (K) & Constant & Key press \\
Keyboard+Alpha (KA) & Alpha power based & Key press \\
Keyboard+Alpha+SSVEP (KAS) & Alpha power based & SSVEP based \\
\hline
\end{tabular}

low and less far if it is high, making the player's mental state control the difficulty of the hunt. Moreover, in an easy game, targets flee less far than in a difficult game where players have to chase their targets with great effort. Therefore in an easy game, movements are generally perceived as slower and in smaller numbers than in a difficult game.

Rules for points for eating targets In all game levels, an unsuccessful eating attempt is penalised with -50 points. In levels $K$ and $K A$, eating is triggered by pressing the Ctrl key. A successful eating attempt rewards the player points proportional to the distance between the centres of the avatar and the closest target. In level $K A S$, approaching a target closer than the threshold radius triggers a SSVEP stimulus appearing next to the target. The association of the stimulus and the target is visualised by a line connecting both. The stimulus consists of a circle with a diameter of 128 pixels and flickers with a frequency of $7.5 \mathrm{~Hz}$, changing from black to white. It is displayed for 6 seconds on the screen. This frequency-stimulus combination was shown to be highly efficient in the elicitation of a SSVEP response [30]. The SSVEP classification results $\left(R\left(f_{H 2, H 3}\right)\right.$, see Sect. 3.2) are recorded within the time interval of 3-6 sec with respect to the stimulus onset (this specific interval depends on the window size of 3 seconds used in the EEG processing). If the mean output of the SSVEP classifier during this inter$\mathrm{val}$ is above 0.5 , the target gets eaten. In this case the player receives points proportional to his mean alpha power measured in the same interval. Thus, for players, it is of benefit to control both alpha band power and SSVEP simultaneously.

\subsection{EEG processing}

The EEG signals were continuously read and processed in 3 -second sliding windows, the interval between window onsets being 1 second. We decided for this specific windowlength, as it gave superior classification results compared to shorter window lengths in conjunction with $7.5 \mathrm{~Hz}$ SSVEP stimulation [30].

Signal processing and machine learning steps were carried out using the Golem and Psychic libraries [42, 43]. The steps for processing a window are depicted in Fig. 3.

A window was first re-referenced using common average referencing (CAR) [26] since no reference electrode was employed during recordings. Then the total alpha power, $P\left(f_{\alpha}\right)$, was obtained by averaging the individual amplitude

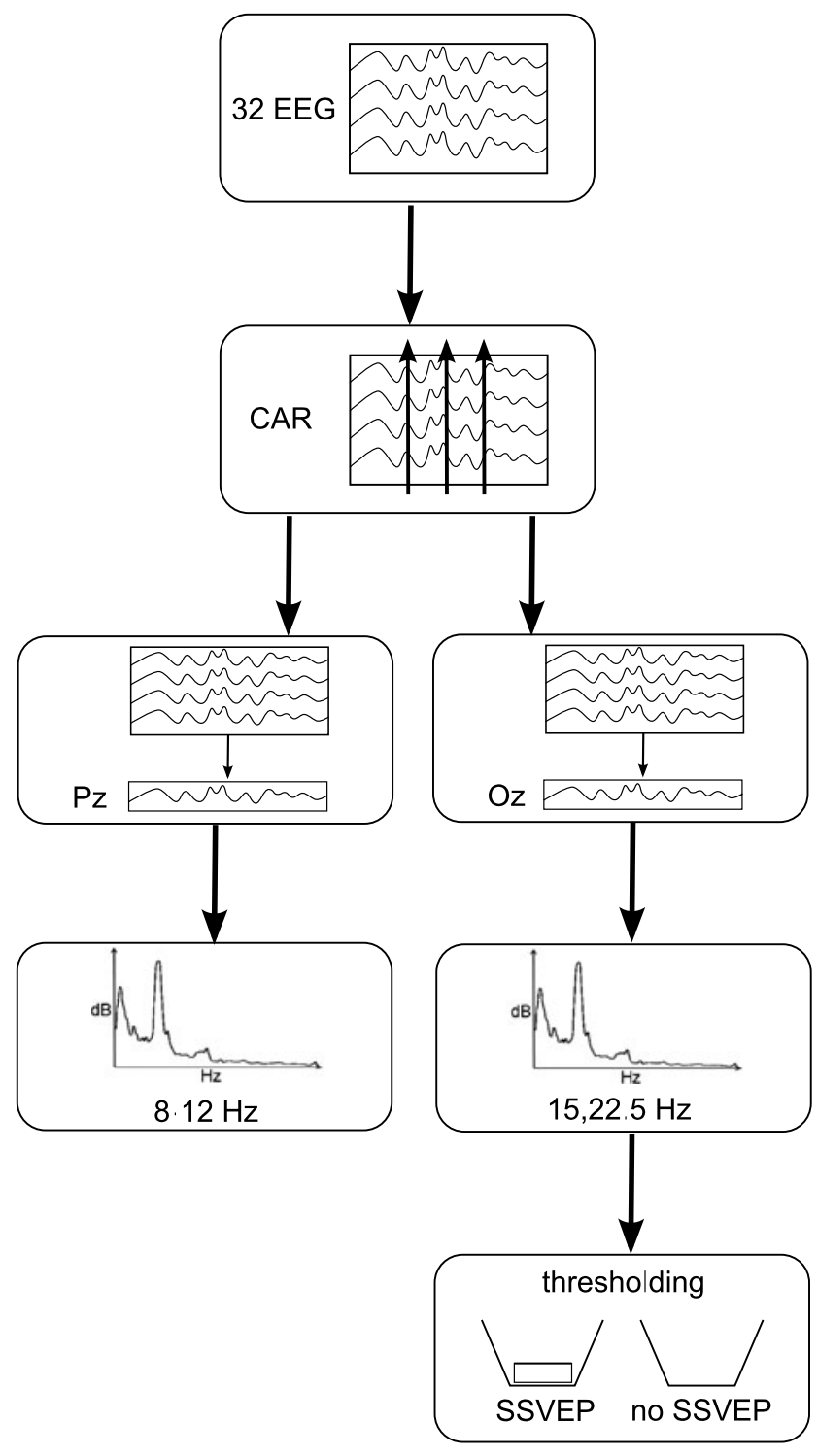

Fig. 3 EEG window processing steps: signal acquisition, common average referencing, channel selection, amplitude computation (FFT) and thresholding for SSVEP detection

values for each integer frequency in $8-12 \mathrm{~Hz}$ at channel $\mathrm{Pz}$ computed by 512-points fast Fourier transform (FFT). For game level $K$, all observed alpha power values $P\left(f_{\alpha}\right)$ were just collected within a vector, $A_{K}$, for use in the next levels and no further processing was done in the window. 
Table 2 Values sent through TCP for each game version

\begin{tabular}{lll}
\hline Level & Alpha power & SSVEP response \\
\hline $\mathrm{K}$ & Constant & Constant \\
$\mathrm{KA}$ & $P_{\text {sca }}\left(f_{\alpha}\right)$ & Constant \\
$\mathrm{KAS}$ & $P_{s c a}\left(f_{\alpha}\right)$ & $R\left(f_{H 2, H 3}\right)$ \\
\hline
\end{tabular}

In games $K A$ and $K A S$, local minima and maxima $\left(\alpha_{\min }\right.$ and $\alpha_{\max }$ ) were defined for normalisation as follows:

$\alpha_{\min }=\mu\left(A_{K}\right)-\left(2 \times \sigma\left(A_{K}\right)\right) \quad$ and

$\alpha_{\max }=\mu\left(A_{K}\right)+\left(2 \times \sigma\left(A_{K}\right)\right)$

where $\mu\left(A_{K}\right)$ and $\sigma\left(A_{K}\right)$ are the mean and standard deviation values of set $A_{K}$. This way, assuming a normal distribution, the interval $\left[\alpha_{\min }, \alpha_{\max }\right]$ would contain $95 \%$ of the cases and eliminate possible outliers [55]. To obtain the final alpha power, $P\left(f_{\alpha}\right)$ was mapped to a value in $[0,1]$ as:

$P_{s c a}\left(f_{\alpha}\right)=\frac{P\left(f_{\alpha}\right)-\alpha_{\min }}{\alpha_{\max }-\alpha_{\min }}$

where $P_{s c a}\left(f_{\alpha}\right)$ is the scaled alpha power value. In case $P_{s c a}\left(f_{\alpha}\right)<0$ and $P_{s c a}\left(f_{\alpha}\right)>1$, it was assigned to 0 and 1 respectively. For game $K A$, window processing ended here.

For game $K A S$, processing went on by SSVEP detection. An adapted version of the detection algorithm by Cheng et al. [7] was implemented for this purpose (see Sect. 3.3 for implementation details and evaluation). Powers in second $(15 \mathrm{~Hz})$ and third $(22.5 \mathrm{~Hz})$ harmonics of the stimulus flickering frequency $(7.5 \mathrm{~Hz})$ at channel $\mathrm{Oz}$ were extracted using 1024-points FFT and averaged. Let $P\left(f_{H 2, H 3}\right)$ denote this average SSVEP response. $P\left(f_{H 2, H 3}\right)$ was standardised as follows:

$z\left(f_{H 2, H 3}\right)=\frac{P\left(f_{H 2, H 3}\right)-\mu\left(f_{10-35}\right)}{\sigma\left(f_{10-35}\right)}$

where $z\left(f_{H 2, H 3}\right)$ is the z-score and $\mu\left(f_{10-35}\right)$ and $\sigma\left(f_{10-35}\right)$ are the mean and standard deviation values for the average power in $10-35 \mathrm{~Hz}$ band. The SSVEP response, $R\left(f_{H 2, H 3}\right)$, was detected with respect to the threshold value of 0.4 , determined as described in the next subsection, as:

$R\left(f_{H 2, H 3}\right)=\left\{\begin{array}{l}1: z\left(f_{H 2, H 3}\right)>0.4 \\ 0: \text { o.w. }\end{array}\right.$

Once the values necessary for a game version were computed out of the window, the values were passed to the game via the TCP connection (see Table 2).

\subsection{SSVEP detection optimisation}

The SSVEP detection algorithm used in the system is based on the algorithm described by Cheng et al. [7]. Their al- gorithm is defined as follows: the SSVEP response is defined as the sum of the power in the fundamental frequency and the power in the second harmonic. This is compared to twice the power of a baseline. In other words, the average of the fundamental frequency and second harmonic has to be higher than the baseline to give a positive classification. The baseline is the mean power in the frequency spectrum between 4 and $35 \mathrm{~Hz}$, determined empirically before the session with real-time feedback. The selection in their system is made in case that a particular option is selected for four consecutive windows. The window size is 512 samples, with a 60-sample inter-window-interval, at a sample frequency of $200 \mathrm{~Hz}$.

This algorithm has been adjusted in a few key ways, in order to make it more robust to spontaneous alpha activity, and personalised without the need for prerecorded data. In time-frequency plots created from the data of the pilot experiments, clear power increases show for the second and third harmonics for most of the subjects, but not always for the fundamental frequency. Based on this information, we decided not to look at the fundamental frequency and second harmonic, but at the second and third harmonics. This means that there is no longer a need to rely on information from the alpha range. As activity in the alpha band is also used as a representation of relaxation which the user can actively try to control or passively perceive, this is a way to separate the two paradigms somewhat. The lower bound of the baseline range has been increased to $10 \mathrm{~Hz}$, in order to make it less susceptible to low-frequency artefacts, for example such caused by eye movements. Whereas Cheng et al. use a fixed baseline, the Bacteria Hunt algorithm first applies z-score normalisation. This is a way to set a subject-independent threshold on previously subjectdependent data, without requiring previous recordings of the subject before starting the session with real-time feedback. It might also make the algorithm more robust to changes over time. Finally, Cheng et al. used electrode O1 and O2, but did not describe how they merged the recordings from these two electrode sites, so we decided to rely for both algorithms on brain activity at the center of the occipital lobe (electrode Oz), where the SSVEP response was expected to be the strongest.

In short, in the Bacteria Hunt algorithm the SSVEP response is defined as the average of the power in the second and third harmonics. In the case of $7.5 \mathrm{~Hz}$ stimulation, this means the average of the power at $15 \mathrm{~Hz}$ and $22.5 \mathrm{~Hz}$. To determine this power a 1024-points fast Fourier transform is applied to the 3-second windows recorded at $512 \mathrm{~Hz}$ sample frequency. This results in a frequency bin size of $0.5 \mathrm{~Hz}$. The SSVEP response is normalised according to the data in the same window, from $10 \mathrm{~Hz}$ to $35 \mathrm{~Hz}$. The mean and standard deviation of power over this frequency range is computed. These values are used for the z-score normalisation. 
Fig. 4 Mean accuracy per threshold, average over the eight datasets from the preliminary experiments. The highest accuracy is yielded with a threshold of 0.4

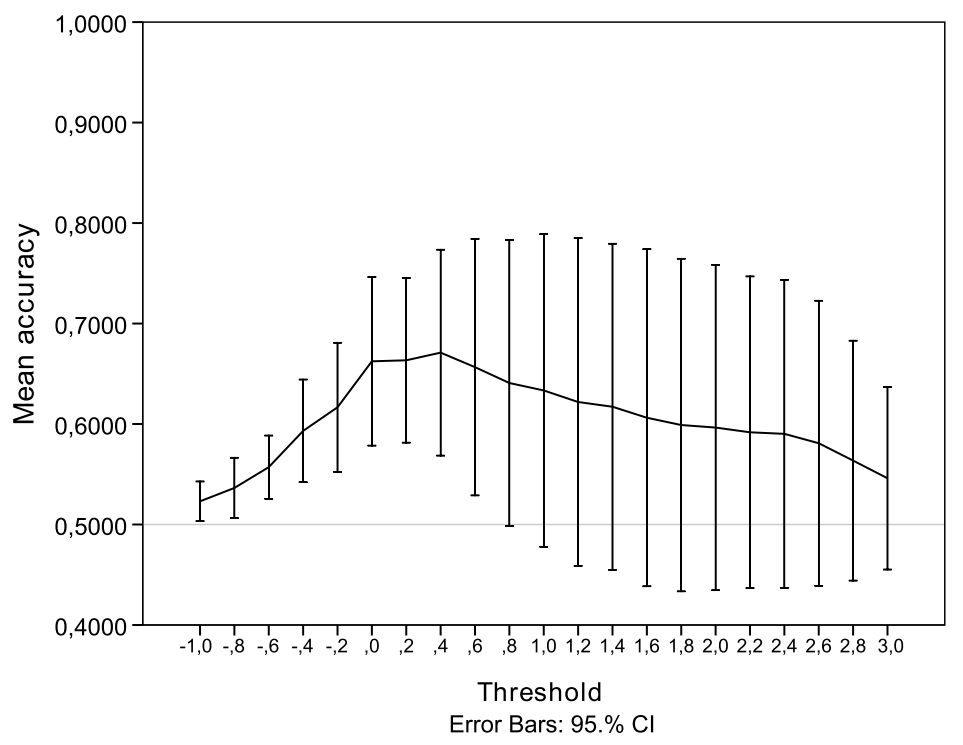

If this normalised response is higher than the fixed threshold, a SSVEP has been detected. This threshold determines the detection accuracy, and therefore has to be estimated carefully.

\subsubsection{Threshold estimation and pilot evaluation}

The threshold estimation was based on eight datasets gathered during preliminary experiments with six subjects. During these pilot studies, subjects played the Bacteria Hunt game, but were forced to have sufficient periods without SSVEP stimulation by waiting to eat a new bacterium for at least six seconds. This way, data was obtained with and without SSVEP stimulation, within the environment later used in the game setting with real-time feedback. Based on this data, the threshold that would result in, on average, the optimal accuracy for SSVEP detection, is 0.4 (Fig. 4). This optimal threshold was used in the main experiment.

In order to decide which of these two classification algorithms, the original or the modified version, should be implemented for the game, the area under the curve (AUC) was used as a performance measure. AUC values are a measure of discriminability of the two classes (SSVEP versus no SSVEP) based on the given values. To be exact: "The AUC of a classifier is equivalent to the probability that the classifier will rank a randomly chosen positive instance higher than a randomly chosen negative instance. This is equivalent to the Wilcoxon test of ranks" [12]. The AUC is based on the receiving-operating characteristics (ROC) curve, which is created by varying the threshold and plotting the resulting true positive rates (hits relative to the total number of windows during which the SSVEP stimulus was shown) and false positive rates (false alarms relative to the total number of windows during which no stimulus was provided). This means on one hand that the class ratio of SSVEP versus no SSVEP has no influence on the resulting ROC or AUC value. Besides, this performance measure is independent of the actually chosen threshold, really focusing on the ability to discriminate on this feature. These are two advantages this measure of performance has over more simple measures such as accuracy (the number of hits and correct rejections relative to the total number of samples). An AUC value of 0.5 would be random performance for a two-class problem. An AUC value of 1.0 would indicate perfect discrimination. From the pilot studies, an average AUC value of 0.64 was computed for the original algorithm of Cheng et al., and of 0.74 for the Bacteria Hunt variety. Although it is based on only those eight datasets, the adaptation of the algorithm is expected to perform better than the original, thus the new algorithm was selected to be used with the game for real-time feedback.

\subsubsection{Evaluation of the SSVEP detection algorithm}

To compare the SSVEP detection algorithm used by Bacteria Hunt with the original developed by Cheng et al., the ROC curve was computed on a superset containing samples from the four game blocks of all nineteen subjects (refer to Sect. 4 for the experiment design). For the samples which are positive for SSVEP stimulation, the brain signal data recorded during the KAS level is epoched starting from one second after the SSVEP stimulation with a duration of three seconds. The samples with no SSVEP stimulation were taken from KA levels, which were simply epoched in subsequent 3 -second windows. The result is a dataset with 1569 windows during SSVEP stimulation, and 4560 windows without. See Fig. 5 for the resulting ROC curves. The AUC value seems slightly larger for the Bacteria Hunt algorithm. 


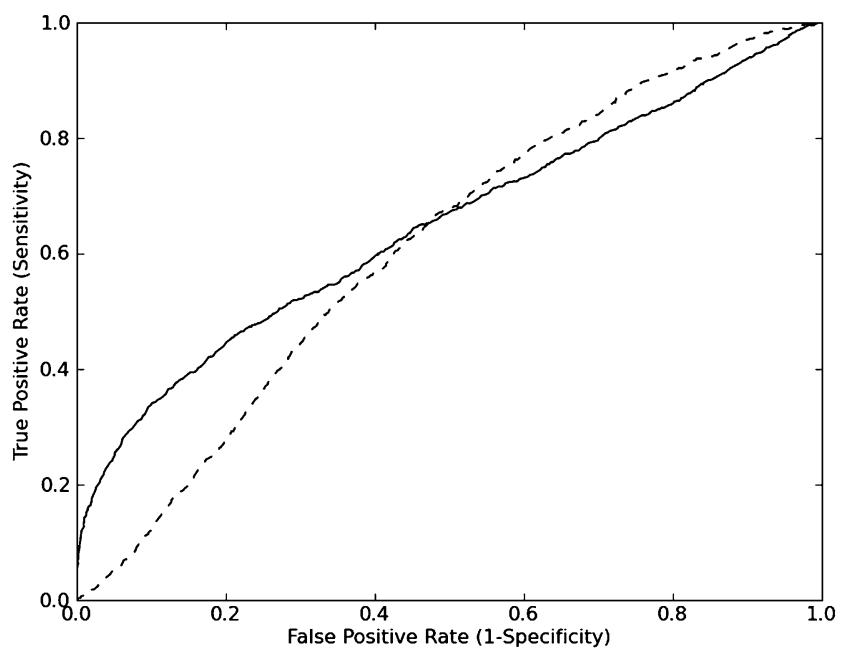

Fig. 5 ROC curves of the SSVEP detection algorithm by Cheng et al. (dashed) and the Bacteria Hunt algorithm. The Bacteria Hunt SSVEP algorithm performs, though not significantly, better overall

However, based on two ROC curves it is not possible to determine whether the difference is significant. Therefore the AUC values per block per subject were examined as well. A paired samples t-test is used to compare the AUC values for the algorithm of Cheng et al. to the Bacteria Hunt version. There is a trend towards a higher performance for Bacteria Hunt $(M=0.65, S D=0.14)$ than Cheng et al. $(M=0.62, S D=0.18)$ with $t(75)=1.775$, $p=0.08$. When comparing the performance for real alpha versus sham alpha blocks, or easy versus difficult games, there is no difference.

A possible problem for the estimation of an optimal threshold is the co-occurrence of SSVEP-unrelated physiological differences during the non-SSVEP trials during gaming. Normal gaming, in contrast to the concentrated fixation during SSVEP, might be contaminated by additional activity occurring due to muscle activity, eye movements and gamerelated cognitive activity. This activity might be found to influence the power in the relevant frequencies, that is between 10 and $35 \mathrm{~Hz}$ and hence bias the SSVEP recognition algorithm, relying on a threshold estimated with relatively clean non-SSVEP trials. Therefore, the conditions during threshold estimation should match the conditions of application, to keep the bias as small as possible.

Summarising, the modified version of Cheng et al. was overall better performing than the original version. An optimal threshold estimation should take the context of use into account, specifically additional activities when no SSVEP stimulus is shown.

\section{Methods}

In the previous section the game and system design were described. In this section we will outline the methods used to study the application of neurofeedback and SSVEP BCI in the context of multimodal gaming.

\subsection{Hypotheses}

The purpose of the experiment was to examine the effect that neurofeedback BCI has on game play and the possible interactions between the paradigms used. The effects were investigated via subjective and objective indicators. Subjective indicators are based on the responses of the subjects to items taken from the Game Experience Questionnaire (GEQ). As objective indicators of the effects of the BCI, we extracted the power in specific frequency bands over the cortical regions of interest and the heart rate (see Sects. 4.2 and 4.6 for details).

To assess the effect that the feedback of the alpha power had, a control condition with sham feedback was devised. We expect the participants to get a better control over their relaxation state in the condition where they actually received feedback of it. This should reflect in their game experience and in the objective indicators of their relaxation, specifically alpha power (see Sect. 2.1) and heart rate [25].

H1a: Neurofeedback leads to less tension, less negative, more positive affect, and a higher feeling of competence.

H1b: Alpha power should increase and the heart rate decrease for the neurofeedback condition.

Furthermore, the interaction of the different BCIs was explored by contrasting the power of alpha before, during, and after the SSVEP stimulation. The alpha rhythm, especially over occipital cortices, is known to be influenced (i.e. decreased) by visual stimulus processing and attention [49]. Despite the spatial separation of the occipital cortex and the site of alpha-recording over the parietal cortex, due to the low spatial resolution of the EEG, the alpha power read might be influenced by the visual stimulus processing.

Besides, the interaction between the two BCI paradigms might also be influenced by the player's tendency to give precedence to the execution of one mental task. As relaxation and concentration are antagonistic mental states most of the time, the BCI paradigms could mutually prevent or limit each other's efficacy. In the KAS level of the game the score for eating is computed using both the SSVEP classification and alpha band power values (see Sect. 3.1.2). However, unless the SSVEP classification is above a threshold, the eating attempt is unsuccessful and the alpha band power is meaningless. Therefore, the players were expected to pay more attention to the SSVEP stimulus than to the relaxation. Thus their alpha band power during the SSVEP attempt would be decreased.

$\mathrm{H} 2$ : SSVEP stimulation leads to a decrease of posterior alpha power measured at parietal electrodes. 
Table 3 The structure of the experiment design, illustrating the combination of the different factors into the four blocks

\begin{tabular}{lll}
\hline & Real $\alpha$ feedback & Sham $\alpha$ feedback \\
\hline Easy game & A & C \\
Difficult game & B & D \\
\hline
\end{tabular}

\subsection{Data acquisition and material}

The game was run on a separate presentation computer (1.8 GHz Pentium M). The EEG signals were recorded with $512 \mathrm{~Hz}$ sample rate via a BioSemi ActiveTwo EEG system and thirty-four $\mathrm{Ag} / \mathrm{AgCl}$ Active electrodes. Additionally, electrocardiogram (ECG), galvanic skin response (GSR), and blood volume pulse (BVP) were recorded. The data was processed and saved on a separate data recording and processing computer (2.53 GHz Quadcore) running BioSemi ActiView software.

To acquire subjective responses from the participants, the game experience questionnaire (GEQ) devised by IJsselsteijn et al. [20] was used. The questionnaire comprises items that are accumulated to 7 scales, each reflecting a specific facet of game experience: competence, immersion, tension, flow, challenge, positive affect, and negative affect. The immersion scale provided by the GEQ was left out of the questionnaire for it's irrelevance in the current study.

\subsection{Participants}

Nineteen participants ( 3 female) took part in the experiment. The mean age was 29 years, ranging from 16 to 47 years. Eighteen participants were right-handed, one was lefthanded. Informed consent was obtained from all subjects. To motivate participants to highest performance levels, and thus to make active use of the advantages a high state of relaxation would bring, two cinema tickets were promised to the participant with the highest score. Six euros per hour or a course credit were given as a reimbursement to the participants.

\subsection{Experiment design}

\subsubsection{Effect of alpha neurofeedback}

In order to test the hypothesis about the influence of neurofeedback, a two-factorial (2 (feedback) $\times 2$ (difficulty)) experimental protocol was devised, resulting in four blocks: easy and real feedback (A), difficult and real feedback (B), easy and sham feedback (C), and difficult and sham feedback (D), as depicted in Table 3.

For the first factor (feedback) the alpha feedback application was manipulated. In the real feedback version an increase of alpha yielded a decrease in mobility of the bacteria.
That is, the successful player would be able to bring the bacteria to a halt, thus scoring more. In the sham feedback version the mobility of the bacteria was controlled by the alpha values from the first of the three games played (level $K$ ). Thus there was no systematic relation between the players instantaneous state and the feedback in the levels $K A$ and $K A S$.

The second factor (difficulty) controlled the strength of the feedback. A high difficulty would mean that the bacteria moved faster and would therefore be harder to catch. This manipulation was introduced to ensure that people's relaxation was not on a ceiling level, obstructing possible increases of relaxation.

Participants were asked to play four game blocks. The order of blocks was pseudorandomised to avoid order effects. Each block contained the 3 games in the following order: $K, K A$, and $K A S$ (see Sect. 3.1.2). Each game lasted for 3 minutes. That made a total duration of 9 minutes per block, excluding small breaks between the games. After each block the participants were given the GEQ questionnaire to evaluate their game experience.

\subsubsection{Interaction between BCI paradigms}

To examine the effect of SSVEP stimulation and the associated concentration on the participant's relaxation and on alpha power, the EEG data segments before, during, and after the SSVEP stimulation in the real alpha neurofeedback condition were of interest. While the user should be relaxed in the periods he is hunting for bacteria (pre-SSVEP and postSSVEP), he should concentrate during the SSVEP stimulation. According to the hypotheses, posterior alpha power should decrease during the SSVEP stimulation periods, relative to its level before and after stimulation. This observation should be clearer in the difficult condition where the bacteria flee more quickly decreasing the possibility of a new SSVEP initiation directly after an SSVEP attempt, and thus preventing overlaps between SSVEP and pre-SSVEP epochs.

\subsection{Procedure}

The participants were seated in front of the computer running the game. The distance to the screen was about 80 $\mathrm{cm}$. They read and signed an informed consent and instructions, and filled in a questionnaire assessing information about the amount of computer usage, prior drug consumption, amount of sleep and so forth. After that the electrode cap was placed and the electrodes connected. The thirty-four electrodes were placed according to the 10-20 system [21] (see Fig. 6). Furthermore, two electrodes were attached to the back of the left and right lower arm of the participant to record ECG (Eindhoven Lead 1 standard). Two GSR electrodes and a plethysmograph, measuring the BVP, were fas- 


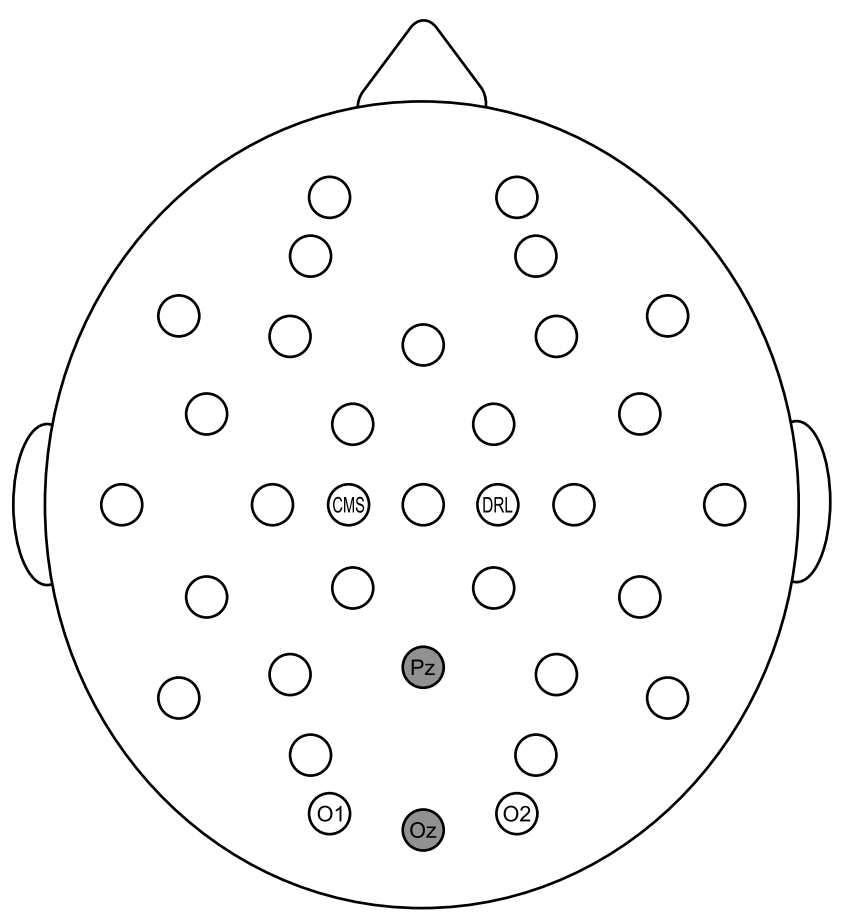

Fig. 6 Electrode locations according to the 10-20 system with Pz and Oz highlighted

tened to the intermediate phalanges of the little and ring fingers, and to the distant phalange of the middle finger of the left hand, respectively.

Immediately before the experiment, the participants were again informed about the differences in control of the 3 games, and specifically the role of alpha power and the possibility to influence it through relaxation.

\subsection{Data analysis}

The answers to the items regarding the participants' experiences during each block of games were accumulated into the 6 scales of the GEQ.

For the analysis of the EEG data, the recordings were processed with EEGlab [9]. After application of a common average reference (CAR), the data was downsampled to $256 \mathrm{~Hz}$, and separated according to the levels and conditions. Then the power of the frequencies of interest was extracted using Welch's method [56], applying windows of 2 seconds. For the overall contrasts of conditions, the data of level $K$ was used as a baseline for levels $K A$ and $K A S$ to remove variance due to frequency differences over time (i.e. blocks) and between participants.

The heart rate extraction was done with the Biosig toolbox [46]. The GSR and BVP recordings were not analysed due to a high amount of movement artefacts.

In order to investigate the interaction between BCI paradigms, difficult and real feedback conditions (block B) of the $K A S$ level recordings for each subject were epoched with

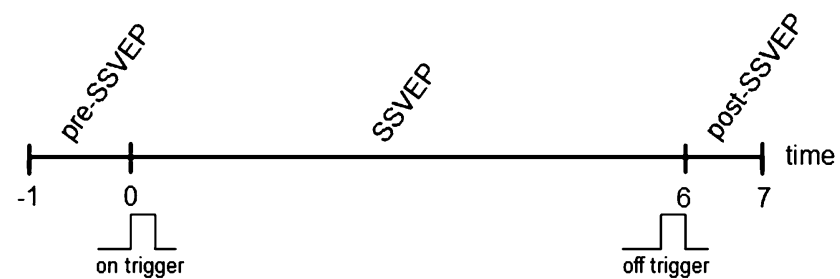

Fig. 7 Epoching with respect to SSVEP triggering

respect to SSVEP stimulus onset and averaged before the extraction of frequency power. The epochs are, as illustrated in Fig. 7, the second of data before the stimulus onset (preSSVEP period), after stimulus onset (SSVEP period) and after stimulus offset (post-SSVEP period).

The Wilcoxon signed-rank test was used for statistical testing of differences between subjective and objective variables, as a violation of the normal distribution was observed for some conditions.

\section{Results}

\subsection{Effects of alpha neurofeedback}

From the post-game questionnaires we extracted the values of the GEQ scales for each subject to examine the effect that the feedback manipulation has on the game experience (see Table 4). Actual feedback of the alpha power was expected to lead to less tension, less negative and more positive affect, and a higher feeling of competence. None of the game experience scales showed significant differences in the feedback contrasts for the easy conditions. However, the difficulty manipulation had the expected effect on game experience. Users felt less competent in their capability to control the game $(p<0.001)$, less relaxed (higher tension) $(p<0.05)$, and more challenged in the difficult conditions of the game $(p<0.01)$. Furthermore, the users felt significantly more positive for the easy real feedback condition $(p<0.01)$, and still showed a trend in the same direction for the sham feedback condition $(p<0.1)$. Nevertheless, the game experience during the difficult conditions did not show significant effects either.

No significant difference between the feedback conditions was found comparing the alpha power values measured at electrode Pz. Also a more exploratory analysis of the alpha band power in the other electrodes did not show reliable effects of neurofeedback. However, an analysis of the development of alpha power over games showed a constant increase of power (see Fig. 8), which is significant for all contrasts of level $K A$ and $K A S(p<0.05)$. This effect is further explored in the following section. Also the analysis of heart rate did not yield significant effects of feedback. Nevertheless, a significant downward trend $(p<0.05)$ for all game levels, except between level $K$ and $K A$ of block $\mathrm{C}$ was found (see Fig. 9). 
Table 4 Mean GEQ scale values (noted as: mean (standard deviation)) for the 4 conditions A (easy real), B (difficult real), C (easy sham), and D (difficult sham), and the p-values for the difficulty and feedback contrasts (bold: significant contrasts)

\begin{tabular}{|c|c|c|c|c|c|c|c|c|}
\hline \multirow{2}{*}{$\begin{array}{l}\text { Name of } \\
\text { Scale }\end{array}$} & \multicolumn{4}{|c|}{ GEQ scale values } & \multicolumn{2}{|c|}{ Difficulty } & \multicolumn{2}{|c|}{ Feedback } \\
\hline & $\bar{A}$ & B & $\mathrm{C}$ & $\mathrm{D}$ & AvsB & CvsD & AvsC & BvsD \\
\hline Competence & $3.3(0.9)$ & $2.3(0.9)$ & $3.2(0.9)$ & $2.5(0.9)$ & 0.000 & 0.000 & 0.810 & 0.242 \\
\hline Flow & $2.9(0.7)$ & $3.0(0.8)$ & $2.9(0.8)$ & $3.0(0.8)$ & 0.563 & 0.611 & 1 & 0.898 \\
\hline Tension & $2.2(0.6)$ & $2.6(0.6)$ & $2.3(0.7)$ & $2.7(0.6)$ & 0.000 & 0.044 & 0.145 & 0.717 \\
\hline Challenge & $2.6(0.5)$ & $3.0(0.5)$ & $2.6(0.5)$ & $2.9(0.6)$ & 0.006 & 0.007 & 0.896 & 0.949 \\
\hline Negative & $2.3(0.7)$ & $2.4(0.8)$ & $2.4(0.8)$ & $2.3(0.8)$ & 0.484 & 0.255 & 0.553 & 0.215 \\
\hline Positive & $3.4(0.7)$ & $3.0(0.8)$ & $3.4(0.8)$ & $3.2(0.7)$ & 0.007 & 0.089 & 0.778 & 0.442 \\
\hline
\end{tabular}

Fig. 8 The development of the alpha power over the games for all four blocks. For better visualization of the power development over the 3 levels no baselining by the power of level $\mathrm{K}$ was applied. The trend towards higher alpha over games might partially be caused by a general tendency of the user to relax over the range of a block

Fig. 9 The development of the heart rate over the games for all four blocks. The data shows a trend towards a lower heart rate, strengthening the interpretation of a general relaxation over the games
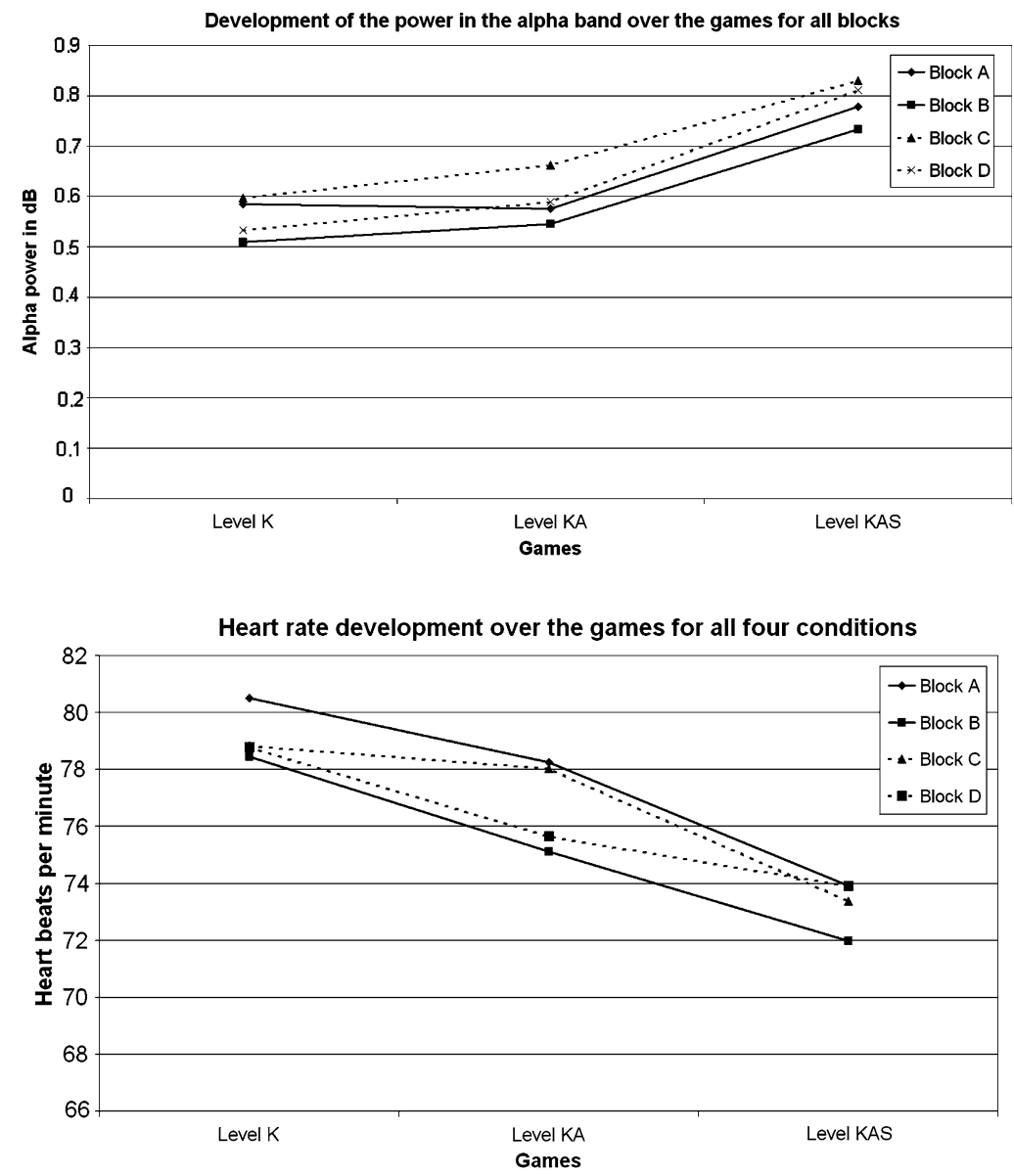

\subsection{Interaction between $\mathrm{BCI}$ paradigms}

The SSVEP epochs were averaged over the trials to inspect the effect of SSVEP on alpha power. The average power in $8-12 \mathrm{~Hz}$ band at channel $\mathrm{Pz}$ was estimated per second. Average alpha power in pre-SSVEP, SSVEP, and post-SSVEP periods were compared to understand the effect of SSVEP stimulation. The alpha power in pre-SSVEP periods was significantly lower $(p<0.05)$ than that in SSVEP and postSSVEP periods. The increase in alpha power during the
SSVEP and post-SSVEP periods could be a reflection of the power increase at the same channel due to the stimulus flickering at $7.5 \mathrm{~Hz}$ frequency, which is very close to the alpha band. The power at $7.5 \mathrm{~Hz}$ at channel Pz during the SSVEP period was also observed to be significantly higher than that in pre-SSVEP and post-SSVEP period. To test the dependence of the effect on the SSVEP stimulation frequency, the analysis was repeated for the frequency band $10-12 \mathrm{~Hz}$ and, indeed, no significant change was observed this time. Table 5 summarises the values. 
Table 5 Mean power spectral densities (in $\mathrm{dB}$ ) for the analysis of the influence of SSVEP stimulation on alpha power

\begin{tabular}{lllll}
\hline Frequency & Channel & Period & & \\
\cline { 3 - 5 } & & Pre-SSVEP & SSVEP & Post-SSVEP \\
\hline $8-12 \mathrm{~Hz}$ & $\mathrm{Pz}$ & 0.034 & 0.050 & 0.052 \\
$10-12 \mathrm{~Hz}$ & $\mathrm{Pz}$ & 0.034 & 0.039 & 0.042 \\
$7.5 \mathrm{~Hz}$ & $\mathrm{Oz}$ & 0.066 & 1.174 & 0.096 \\
\hline
\end{tabular}

\section{Discussion}

A multimodally controlled multi-paradigm BCI game was designed and implemented [30] to test the simultaneous use of several BCI paradigms in addition to traditional input in one game. One goal of the current study was to examine the effect of neurofeedback on the ability of the user to manipulate his mental state (i.e. relaxation) to control an aspect of the game world (i.e. the bacteria's flight speed). A second goal was the identification of possible interactions between the two BCI paradigms employed within the game. Additionally, the novel approach to SSVEP detection is discussed.

\subsection{Effects of alpha neurofeedback}

The comparison of real and sham feedback conditions did not provide evidence for the expected effects of the neurofeedback. Neurofeedback did not affect game experience, heart rate, or the target frequency significantly. The physiological effects were also expected on the basis of clinical feedback studies, which show that people are able to influence their brain activity when it is made perceivable $[15,28]$. In the following section we discuss methodological and technical issues that might have led to these results.

Studies on (generalised) anxiety in patients and nonpatients show that alpha neurofeedback (i.e. alpha enhancement training) can decrease the level of stress and anxiety $[10,16,17,44]$. Although the current study's rationale and methodology differ from the traditional neurofeedback research, some important lessons might be learned from it.

One of the most important factors of the efficacy of neurofeedback seems to be the amount of training participants receive [28]. Neurofeedback therapy is mostly done over several sessions [3], though physiological effects of neurofeedback can already be observed after one session [13, 31].

Furthermore, an important difference of our study to therapeutic neurofeedback studies was that the feedback was not given as a discrete signal of success or failing to enhance alpha [31], but was visualised continuously. This might attenuate the saliency of the feedback and consequently of the reward. By increasing the game difficulty this relationship was made more salient, however, without success.
Another problematic issue is the high level of relaxation in the subject population (students), possibly leading to a floor effect of relaxation. Hardt and Kamiya [16] observed an effect of NF therapy on students with high levels of anxiety, but not on those with low levels. Therefore it is possible that the original level of relaxation was not further attenuable by a neurofeedback procedure. A careful selection of a highly stressed test population would be advised to evaluate the efficacy of a neurofeedback system. In the current experiment we tried to induce a higher level of stress via the more difficult game level. This did not result in an effect, despite the successful increase of the stress level.

Moreover, if the participants were relaxing as much as possible all the time they might not be able to associate changes of their state to changes in feedback, rendering the feedback manipulation ineffective. To avoid this, game dynamics could be changed to encourage the user to relax at certain moments and tense up at others.

The technical implementation of a feedback system and the choice of parameters for the extraction of the physiological features are further relevant issues.

The short three-second window for the analysis of alpha power is very susceptible to spontaneous changes in alpha power. A longer EEG window would have attenuated these spontaneous fluctuations in the feedback and thereby strengthened its relation to relaxation.

Moreover, common average reference was used in the EEG processing steps, as it has been shown to be superior to other referencing methods in a BCI context [26]. This technique, however, might also lead to the cancellation of globally synchronous activity. It thereby might possibly cancel out changes in global alpha activity related to relaxation $[5,6]$. Alternatively, the linked-ears reference could be used [27].

Eye movements can cause additional variance in the alpha frequency band. To attenuate their influence an option would be to detect and remove eye movements from the data automatically [47].

Finally, alpha power might vary with several factors other than relaxation. It is for example sensitive to the opening and closing of the eyelid, but it is also implied in several cognitive processes, like working memory and sensory processing $[24,37,48]$. This might introduce an additional source of variance in the alpha power, especially in complex tasks. A dedicated experiment should validate the role of alpha power as predictor for relaxation in a complex natural $\mathrm{HCI}$ scenario.

Summarising, it might be doubted that the general approach of neurofeedback-guided relaxation during engaged computer gaming is feasible. However, it was shown in the very simple biofeedback game "Brainball" that relaxation (and stress) derived from physiological sensors can be part of the game dynamic and in the same time affected by it [19]. 
Above several issues were highlighted that might partially or in the whole be responsible for the inability to show an effect of neurofeedback in a more complex and multimodal gaming environment. Addressing these issues could lead to the isolation of factors that hinder the successful application of neurofeedback BCI in complex gaming scenarios, and thereby enable the design of effective neurofeedback BCIs for multimodal games.

\subsection{Interactions between the BCI paradigms}

The analysis done to investigate the influence of BCI paradigms on each other revealed that activity in a certain frequency band, at a certain electrode site can affect the activity in a nearby frequency interval, at a spatially close electrode site. Thus, while designing BCIs, one should consider keeping different paradigms far from each other in terms of frequency and electrode location to prevent undesired interferences.

The constant trend of high parietal alpha band power before, during, and after the SSVEP interval indicates that, despite a potential blocking effect of the SSVEP on the alpha rhythm, subjects were persistent in maintaining their alpha power in compliance with the relaxation task as they were instructed.

\subsection{Evaluation of the SSVEP detection algorithm}

In Sect. 3.3 we motivated and evaluated a modification of the SSVEP detection algorithm introduced by Cheng et al. [7]. There is a trend indicating that the modified Bacteria Hunt SSVEP algorithm has a better detection performance than that of Cheng. This might be due to the fact that often the fundamental frequency of the SSVEP stimulation is not as clearly increasing during stimulation as its harmonics (see also Jia et al. [22]). Our modified algorithm uses only the second and third harmonics to attenuate this problem.

\section{Conclusion}

In this study a game was used to investigate the interaction with BCIs in a natural environment. The goal of the study was twofold.

Firstly, the aim was to show the efficacy of alpha-based neurofeedback. Sham feedback was used to examine the effect that the perceived control over the BCI has on the game experience. It was expected that real feedback would subjectively lead to a more relaxed state and greater feeling of confidence. Furthermore, differences in objective indicators of relaxation were expected. The analysis showed no difference in subjective and objective indicators of relaxation or control. Several possible methodological and technical factors for the inefficacy of the neurofeedback were identified.
Future work should try to evaluate their role to enable a successful alpha neurofeedback-based BCI.

Secondly, the interaction between different, simultaneously used BCI paradigms, namely alpha neurofeedback and SSVEP, were studied. Due to the antagonistic nature of these two mental tasks, relaxation and focused attention, and due to the blockage of the alpha rhythm during visual stimulation, a decrease of alpha was expected during SSVEP. However, an increase in alpha power was found. Further analysis suggested that the increase was due to the increase of the power in the first harmonic of the SSVEP stimulation $(7.5 \mathrm{~Hz})$, which is close to the alpha band $(8-12 \mathrm{~Hz})$. Careful examination of possible influences of multiple BCI paradigms on each other is advised.

Furthermore, a novel SSVEP detection algorithm was developed and compared against a standard method. The advantage over the standard method is an independence of prior baseline recordings. The viability of the new approach was shown by evaluation results comparable to the standard method.

Acknowledgements This research has been supported by the GATE project, funded by the Netherlands Organization for Scientific Research (NWO) and the Netherlands ICT Research and Innovation Authority (ICT Regie), and by the BrainGain Smart Mix Programme of the Netherlands Ministry of Economic Affairs and the Netherlands Ministry of Education, Culture and Science.

The authors would like to thank Boris Reuderink for his help with the EEG processing and Marijn van Vliet for the icons used in Fig. 3.

Open Access This article is distributed under the terms of the Creative Commons Attribution Noncommercial License which permits any noncommercial use, distribution, and reproduction in any medium, provided the original author(s) and source are credited.

\section{References}

1. Aarseth E (2003) Playing research: methodological approaches to game analysis. In: Proceedings of the 5th international digital arts and culture conference (DAC), Melbourne, Australia

2. Allison BZ, McFarland DJ, Schalk G, Zheng SD, Jackson MM, Wolpaw JR (2008) Towards an independent brain-computer interface using steady state visual evoked potentials. Clin Neurophysiol 119(2):399-408

3. Ancoli S, Kamiya J (1978) Methodological issues in alpha biofeedback training. Appl Psychophysiol Biofeedback 3(2):159183

4. Arns M, de Ridder S, Strehl U, Breteler M, Coenen A (2009) Efficacy of neurofeedback treatment in ADHD: the effects on inattention, impulsivity and hyperactivity: a meta-analysis. Clin EEG Neurosci 40(3): 180-189

5. Barry RJ, Clarke AR, Johnstone SJ, Magee CA, Rushby JA (2007) EEG differences between eyes-closed and eyes-open resting conditions. Clin Neurophysiol 118(12):2765-2773

6. Barry RJ, Clarke AR, Johnstone SJ, Brown CR (2009) EEG differences in children between eyes-closed and eyes-open resting conditions. Clin Neurophysiol 120(10):1806-1811

7. Cheng M, Gao X, Gao S, Xu D (2002) Design and implementation of a brain-computer interface with high transfer rates. IEEE Trans Biomed Eng 49(10):1181-1186 
8. Cohen BA, Sances A Jr (1977) Stationarity of the human electroencephalogram. Med Biol Eng Comput 15(5):513-518

9. Delorme A (2004) EEGLAB: an open source toolbox for analysis of single-trial EEG dynamics including independent component analysis. J Neurosci Methods 134(1):9-21

10. Egner T, Gruzelier JH (2003) Ecological validity of neurofeedback: modulation of slow wave eeg enhances musical performance. Neuroreport 14(9):1221-1224

11. Fairclough SH (2009) Fundamentals of physiological computing. Interact Comput 21(1-2):133-145

12. Fawcett $T$ (2006) An introduction to ROC analysis. Pattern Recogn Lett 27(8):861-874

13. Fell J, Elfadil H, Klaver P, Röschke J, Elger CE, Fernández G (2002) Covariation of spectral and nonlinear EEG measures with alpha biofeedback. Int J Neurosci 112(9):1047-1057

14. Gruzelier J, Egner T, Vernon D (2006) Validating the efficacy of neurofeedback for optimising performance. Prog Brain Res 159:421-431

15. Hammond D (2005) Neurofeedback treatment of depression and anxiety. J Adult Develop 12(2):131-137

16. Hardt JV, Kamiya J (1978) Anxiety change through EEG alpha feedback seen only in high anxiety subjects. Science 201(201): 79-81

17. Hare JF, Timmons BH, Roberts JR, Burman AS (1982) EEG alpha-biofeedback training: an experimental technique for the management of anxiety. J Med Eng Technol 6(1):19-24

18. Herrmann C (2001) Human EEG responses to $1-100 \mathrm{~Hz}$ flicker: resonance phenomena in visual cortex and their potential correlation to cognitive phenomena. Exp Brain Res 137(3):346-353

19. Hjelm SI (2003) Research + design: the making of brainball. Interactions 10(1):26-34

20. IJsselsteijn W, de Kort Y, Poels K, Jurgelionis A, Bellotti F (2007) Characterising and measuring user experiences in digital games. In: International conference on advances in computer entertainment technology (ACE 2007), Salzburg, Austria

21. Jasper HH (1958) The ten-twenty electrode system of the international federation. Electroencephalogr Clin Neurophysiol 10:371375

22. Jia C, Xu H, Hong B, Gao X, Zhang Z, Gao S (2007) A human computer interface using SSVEP-based BCI technology. In: Schmorrow DD, Reeves LM (eds) Foundations of augmented cognition, lecture notes in computer science, vol 4565. Springer, Berlin, Heidelberg, pp 113-119, Chap 13

23. Kelly SP, Lalor EC, Finucane C, McDarby G, Reilly RB (2005) Visual spatial attention control in an independent brain-computer interface. IEEE Trans Bio-Medical Eng 52(9):1588-1596

24. Klimesch W (1999) EEG alpha and theta oscillations reflect cognitive and memory performance: a review and analysis. Brain Res Rev 29(2-3):169-195

25. Lang PJ, Greenwald MK, Bradley MM, Hamm AO (1993) Looking at pictures: Affective, facial, visceral, and behavioral reactions. Psychophysiology 30(3):261-273

26. McFarland DJ, McCane LM, David SV, Wolpaw JR (1997) Spatial filter selection for EEG-based communication. Electroencephalogr Clin Neurophysiol 103(3):386-394

27. Miller G, Lutzenberger W, Elbert T (1991) The linked-reference issue in EEG and ERP recording. J Psychophysiol 5:273

28. Moore N (2005) The neurotherapy of anxiety disorders. J Adult Develop 12(2):147-154

29. Morgan ST, Hansen JC, Hillyard SA (1996) Selective attention to stimulus location modulates the steady-state visual evoked potential. Proc Nat Acad Sci USA 93(10):4770-4774

30. Mühl C, Gürkök H, Plass-Oude Bos D, Thurlings ME, Scherffig L, Duvinage M, Elbakyan AA, Kang S, Poel M, Heylen D (2010) Bacteria Hunt: a multimodal, multiparadigm BCI game. In: Proceedings of the 5 th international summer workshop on multimodal interfaces (eNTERFACE’09), Genova, Italy, pp 41-62
31. Mulholland T, Goodman D, Boudrot R (1983) Attention and regulation of EEG alpha-attenuation responses. Appl Psychophysiol Biofeedback 8(4):585-600

32. Müller MM, Picton TW, Valdes-Sosa P, Riera J, Teder-Sälejärvi WA, Hillyard SA (1998) Effects of spatial selective attention on the steady-state visual evoked potential in the $20-28 \mathrm{~Hz}$ range. Brain Res Cogn Brain Res 6(4):249-261

33. Müller-Putz G, Scherer R, Brauneis C, Pfurtscheller G (2005) Steady-state visual evoked potential (SSVEP)-based communication: impact of harmonic frequency components. J Neural Eng 2(4):123-130

34. Nijholt A, Tan D, Pfurtscheller G, Brunner C, Millán JDR, Allison B, Graimann B, Popescu F, Blankertz B, Müller KR (2008) Braincomputer interfacing for intelligent systems. IEEE Intell Syst, pp 76-83

35. Nijholt A, Reuderink B, Plass-Oude Bos D (2009) Turning shortcomings into challenges: brain-computer interfaces for games. In: Intelligent technologies for interactive entertainment, pp 153-168

36. Nowlis DP, Kamiya J (1970) The control of electroencephalographic alpha rhythms through auditory feedback and the associated mental activity. Psychophysiology 6(4):476-484

37. Palva S, Palva J (2007) New vistas for $\alpha$-frequency band oscillations. Trends Neurosci 30(4):150-158

38. Pfurtscheller G, Lopes da Silva FH (1999) Event-related EEG/MEG synchronization and desynchronization: basic principles. Clin Neurophysiol 110(11):1842-1857

39. Plass-Oude Bos D, Reuderink B, Laar B, Gürkök H, Mühl C, Poel M, Nijholt A, Heylen D (2010) Brain-computer interfacing and games. In: Tan DS, Nijholt A (eds) Brain-computer interfaces. Springer, London, pp 149-178, Chap 10

40. Regan D (1989) Human brain electrophysiology: Evoked potentials and evoked magnetic fields in science and medicine. Appleton \& Lange, East Norwalk

41. Reuderink B (2008) Games and brain-computer interfaces: the state of the art. Tech rep, Human-Media Interaction, University of Twente, The Netherlands

42. Reuderink B (2009a) golemml: Python machine learning library for EEG processing. http://code.google.com/p/golemml

43. Reuderink B (2009b) psychicml: Python signal processing library for EEG processing. http://code.google.com/p/psychicml

44. Rice KM, Blanchard EB, Purcell M (1993) Biofeedback treatments of generalized anxiety disorder: preliminary results. Appl Psychophysiol Biofeedback 18(2):93-105

45. Scherer R, Schloegl A, Lee F, Bischof H, Janša J, Pfurtscheller G (2007) The self-paced Graz brain-computer interface: methods and applications. Comput Intell Neurosci

46. Schlögl A, Brunner C (2008) Biosig: A free and open source software library for BCI research. Computer 41(10):44-50

47. Schlögl A, Keinrath C, Zimmermann D, Scherer R, Leeb R, Pfurtscheller G (2007) A fully automated correction method of EOG artifacts in EEG recordings. Clin Neurophysiol 118(1): 98-104

48. Schürmann M, Başar E (2001) Functional aspects of alpha oscillations in the EEG. Int J Psychophysiol 39(2-3):151-158

49. Shaw JC (1996) Intention as a component of the alpha-rhythm response to mental activity. Int J Psychophysiol 24(1-2):7-23

50. Skidmore T, Hill H (1991) The evoked potential human-computer interface. Proc Ann Int Conf IEEE Eng Med Biol Soc 13(1): 407-408

51. Sokhadze TM, Cannon RL, Trudeau DL (2008) EEG biofeedback as a treatment for substance use disorders: review, rating of efficacy and recommendations for further research. J Neurother: Invest Neuromodulation Neurofeedback Appl Neurosci 12(1):5-43

52. Sterman M, Egner T (2006) Foundation and practice of neurofeedback for the treatment of epilepsy. Appl Psychophysiol Biofeedback 31(1):21-35 
53. Thurlings ME, Brouwer AM, van Erp JBF, Werkhoven PJ (2009) SSVEPs for BCI? The effect of stimulus eccentricity on SSVEPs, abstract presented at the 39th Annual Meeting of the Society for Neuroscience

54. Thurlings ME, Erp JBF, Brouwer AM, Werkhoven PJ (2010) EEG-based navigation from a human factors perspective. In: Tan DS, Nijholt A (eds) Brain-computer interfaces. Springer, London, pp 71-86, Chap 5

55. Ward A, Murray-Ward M (1999) Assessment in the classroom. Wadsworth, Belmont
56. Welch P (1967) The use of fast Fourier transform for the estimation of power spectra: a method based on time averaging over short modified periodograms. IEEE Trans Audio Electroacoust 15(2):70-73

57. Zander TO, Kothe C, Jatzev S, Gaertner M (2010) Enhancing human-computer interaction with input from active and passive brain-computer interfaces. In: Tan DS, Nijholt A (eds) Braincomputer interfaces. Springer, London, pp 181-199, Chap 11 